\title{
Vector Autoregressive Modelling of Fire Signals
}

\author{
ALEXANDER FISCHER and HEINZ LUCK \\ Duisburg University \\ Department of Communication Engineering \\ Bismarckstr. 81, 47057 Duisburg, Germany
}

\begin{abstract}
The application of multi sensor detectors and/or sophisticated signal processing systems are two of the most important developments in fire detection technology. Due to the increasing complexity involved the layout of fire detection algorithms becomes more and more elaborate. Based on a representative set of recorded sensor signals layout procedures and performance studies of fire detection algorithms can be aided by simulation techniques. This requires adequate and efficient modelling of the signal recordings by a stochastic model. This paper presents a modelling technique based on a vector autoregressive (VAR) model. This model takes into account the cross correlations between the single sensor signals and is therefore well applicable in the field of automatic fire detection. It can serve as a basic component of a development tool for the design of detection algorithms in automatic fire detection technology. The applicability of the model will be shown by simulation results for signals of a ionization chamber, an optical scattered light. smoke sensor and temperature sensor used for automatic fire detection measurements in the field.
\end{abstract}

KEYWORDS: automatic fire detection, vector autoregressive model, multi sensor detection algorithms 


$\begin{aligned} \overleftrightarrow{a}_{i} & \text { VAR model parameter } \\ b & \text { postprocessing integration constant } \\ D(t) & \text { detection quantity } \\ \vec{e}(t) & \text { vector white noise process } \\ k_{\mathrm{S}} & \text { spike tolerance factor } \\ L & \text { length of signal vector } \\ L_{\mathrm{S}} & \text { spike detection window length } \\ L_{\mathrm{R}} & \text { mean value interpolation interval } \\ L_{\mathrm{T}} & \text { threshold detector parameter } \\ n & \text { number of channels } \\ p & \text { model order } \\ p_{\max } & \text { maximum model order } \\ \vec{r}_{x x}(\tau) & \text { autocorrelation matrix of lag } \tau \\ S(t) & \text { spike detection quantity } \\ S_{\mathrm{T}} & \text { detection threshold }\end{aligned}$

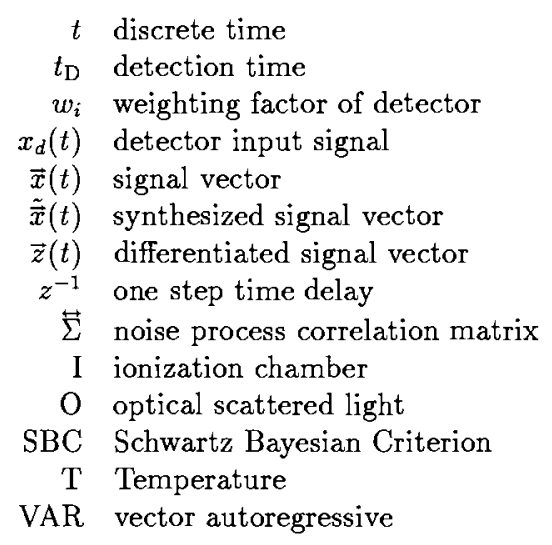

\section{INTRODUCTION}

In the past and to great extent even now automatic fire detection systems consist of sensors known to be useful in this area (ionization chamber, optical scattered light smoke sensor and temperature sensor) with more or less simple signal processing and detection principles. These systems have proved their capability to detect fires in practical applications. But with the increasing number of installations the problem of unwanted alarms becomes more and more apparant. In extreme cases fire brigades had to reject any further connections to automatic fire detection systems because they were mainly serving unwanted alarms. An unwanted alarm is any alarm given by a fire detection system when there is no fire. This may be due to failures of some component in the detection systems, misuse by personel or a misinterpretation of the current environmental condition based on the monitored signal(s). To address this problem (especially the case of misinterpretation) fire detection systems equipped with multiple different sensors linked together for the fire detection purpose have emerged. The use of more than one sensor provides additional information on the environmental condition and allows a less erroneous distinguishment between the fire and no-fire case.

The layout of multi-sensor detectors with signal processings beyond threshold detection and alarm delay offers lots of degrees of freedom. The problem of parameter tuning and comparative studies between different principles can be carried out practically only in the fire case. So as in many other areas there is the need for a simulation technique for studying multi-sensor fire detection algorithms. We will formulate a model for the signals of common sensors in automatic fire detection technology and thereby provide the basis for a simulation tool for the design and study of multi-sensor fire detection algorithms.

The paper is organized as follows. First the theory of the VAR signal model will be discussed. Next the the necessary extensions to apply the VAR model to the modelling of 


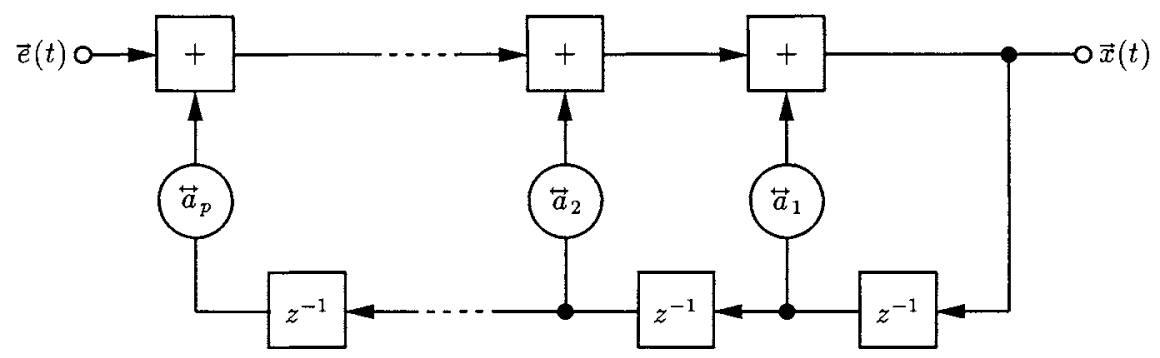

FIGURE 1: Block diagram of the VAR(p)-model

fire signals will be introduced. Then simulation results for an example study of the performance of a multi-sensor based fire detection algorithm will be given. A short conclusion with perspectives for future work finishes the paper.

\section{THEORY OF THE VAR SIGNAL MODEL}

The vector autoregressive (VAR) model is defined by

$\vec{x}(t)=\vec{e}(t)+\sum_{i=1}^{p} \vec{a}_{i} \vec{x}(t-i)$

with the matrices $\vec{a}_{i}, i=, \ldots, p$ being the model parameters, $p$ the model order and $\{\vec{e}(t)\}$ a multichannel stationary white noise process distributed as $\mathrm{N}(\overrightarrow{0}, \vec{\Sigma})$. The signal vector $\vec{x}(t)=\left[x_{1}(t), x_{2}(t), \ldots, x_{n}(t)\right]^{\mathrm{T}}$ at time instant $t$ has the $n$ signals from the $n$ sensors $x_{j}(t), j=1, \ldots, n$ as components. This vector signal model with matrices as parameters allows the adequate description of correlated sets of signals since it takes into account these intercorrelations through the non-diagonal matrix components.

For a set of signal vectors $\vec{x}(t)$, recorded from $t=0, \ldots, L-1$ the task is now to estimate the model parameters $\overleftrightarrow{a}_{i}$, the model order $p$ and the residual correlation matrix of the white noise process $\stackrel{\leftrightarrow}{\Sigma}$.

The so called YULE-WALKER equations for the VAR-model are [1], [2], [3]

$$
\left[\begin{array}{cccc}
\overleftrightarrow{r}_{x x}(0) & \overleftrightarrow{r}_{x x}(1) & \ldots & \overleftrightarrow{r}_{x x}(p-1) \\
\overleftrightarrow{r}_{x x}(1) & \vec{r}_{x x}(0) & \ldots & \overleftrightarrow{r}_{x x}(p-2) \\
\vdots & & \ddots & \vdots \\
\overleftrightarrow{r}_{x x}(p-1) & \overleftrightarrow{r}_{x x}(p-2) & \ldots & \overleftrightarrow{r}_{x x}(0)
\end{array}\right]\left[\begin{array}{c}
\overleftrightarrow{a}_{1} \\
\overleftrightarrow{a}_{2} \\
\vdots \\
\overleftrightarrow{a}_{p}
\end{array}\right]=\left[\begin{array}{c}
\overleftrightarrow{r}_{x x}(1) \\
\overleftrightarrow{r}_{x x}(2) \\
\vdots \\
\overleftrightarrow{r}_{x x}(p)
\end{array}\right]
$$


The residual correlation matrix $\overleftrightarrow{\Sigma}$ of the white noise process is given by

$\overleftrightarrow{\Sigma}=\overleftrightarrow{r}_{x x}(0)-\sum_{i=1}^{p} \vec{a}_{i} \overleftrightarrow{r}_{x x}(i)$

To estimate the parameters of a VAR model the correlation matrices $\vec{r}_{x x}(\tau)$ in $(2)$ and (3) are replaced by their estimated counterparts $\hat{\vec{r}}_{x x}(\tau)$ from a set of $L$ signal vectors $\vec{x}(t), t=0, \ldots, L-1$ according to

$\hat{\vec{r}}_{x x}(\tau)=\frac{1}{L} \sum_{t=\tau}^{L-1}\left[\begin{array}{ccc}x_{1}(t) x_{1}(t-\tau) & \ldots & x_{1}(t) x_{n}(t-\tau) \\ \vdots & \ddots & \vdots \\ x_{n}(t) x_{1}(t-\tau) & \ldots & x_{n}(t) x_{n}(t-\tau)\end{array}\right] \quad, \quad \tau=0, \ldots, p$

The model order can be determined based on a set of $p_{\max }$ estimated models for $p=$ $1, \ldots, p_{\max }$ and the selection of an "optimal" order by the use of Schwartz's Bayesian Criterion [4]

$\operatorname{SBC}(p)=\ln \left|\hat{\Sigma}_{p}\right|+n^{2} p \frac{\ln L}{L}$

$\hat{\vec{\Sigma}}_{p}$ denotes the estimated residual correlation matrix for a model of order $p$. The optimal model order is the one which minimizes $\operatorname{SBC}(p)$ in $p=\left[1, p_{\max }\right]$.

With equation (2) to (5) the estimation of a VAR $(p)$-model including the model order can be achieved for a set of signal vectors $\vec{x}(t), t=0, \ldots, L-1$ assumed to be stationary. In general and in most practical situations this stationarity assumption will not be fulfilled. But in most applications signal characteristics will not change drastically from one time step to another so that blocks in the signal recordings can be defined, which can be described by stationary signal models. Once these blocks (segments) have been identified the above model estimation procedure can be applied to each of the identified segments. This segmentation procedure can be performed with the General Likelihood Ratio (GLR) algorithm by APPEL and BRANDT [5] extended to the multichannel case by KLOSE [6].

Due to the possible numerical problems and computational load being involved with the block matrix inversion in (2) the application of algorithms circumventing these disadvantages is advisable. The First Splitted Generalized LeRoux-Gueguen-algorithm for the multichannel case of SoKAT [7] is a time- and order-recursive algorithm for the solution of (2) in the time-variant case. The order-recursive feature is especially helpful for the order estimation problem since the estimation of a model of order $p_{\max }$ produces all necessary information $\left(\hat{\vec{\Sigma}}_{p}\right)$ for the lower order models. So the algorithm has to be run only once even for the order estimation. 

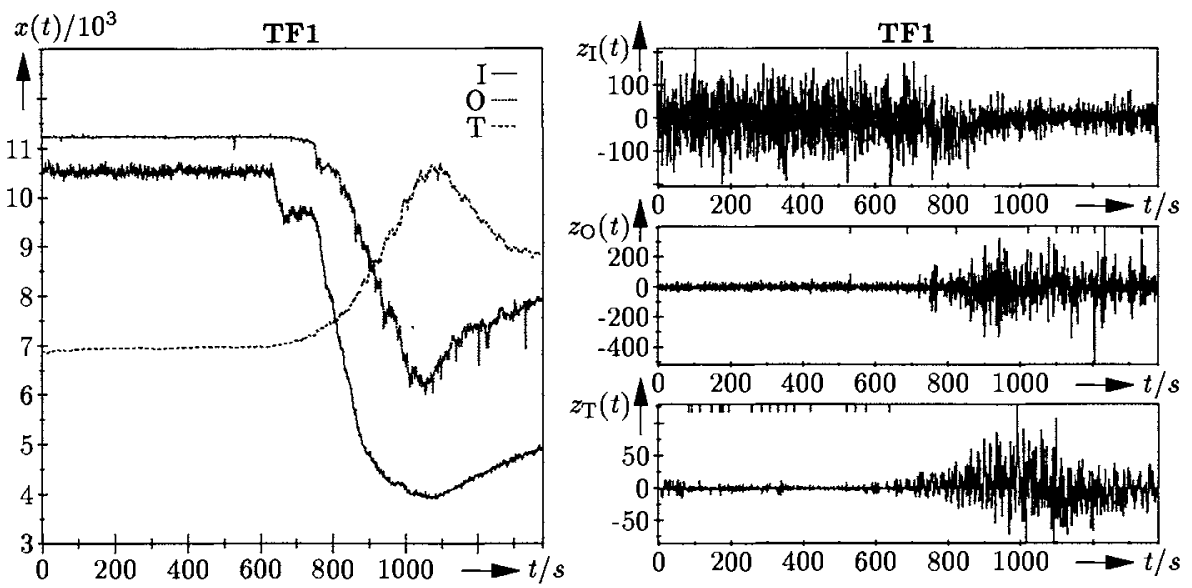

FIGURE 2: Original and preprocessed $\left(L_{\mathrm{S}}=30\right.$ and $\left.k_{\mathrm{S}}=3\right)$ signals $(\mathrm{I}, \mathrm{O}$, and $\mathrm{T})$ for EN $54 / 9$ test fire 1 . The position of detected spikes is indicated by small vertical lines at the top of the right windows.

\section{APPLICATION TO FIRE SIGNAL MODELING}

Figure 2 left shows a set of three recordings of sensors used in fire detectors $x_{\mathrm{I}}(t), x_{\mathrm{O}}(t)$ and $x_{\mathrm{T}}(t)$ corresponding to the measurements of an ionization chamber (I), an optical scattered light smoke sensor $(\mathrm{O})$ and temperature sensor $(\mathrm{T})$ during a test fire $\mathrm{TF} 1$ according to EN 54 part 9 [8].

Because of the properties of the white noise process $\{\vec{e}(t)\}$ in (1) the VAR model is only capable to model signals with zero mean. Obviously this is not the case for the above shown fire signals. So the signals have to be preprocessed before subjecting them to the model estimation procedure. The preprocessing should cover the following two aspects:

1. The preprocessed signals should have zero mean.

2. Signal characteristics strongly disturbing the model estimation should be eliminated. The averaging in (4) over a time interval of length $L$ is strongly influenced by single large positive or negative signal values (spikes).

The applied preprocessing reduces the mean to zero by differentiating and clips each resulting signal value to $k_{\mathrm{S}}$ times the standard deviation determined over a surrounding of length $L_{\mathrm{S}}$ around the actual signal value.

$$
\begin{aligned}
& z_{j}(t)=x_{j}(t)-x_{j}(t-1) \\
& z_{j}(t)=\left\{\begin{array}{cl}
\operatorname{sign}\left(z_{j}(t)\right) k_{\mathrm{S}} S(t) & \text { if }\left|z_{j}(t)\right|>k_{\mathrm{S}} S(t) \\
z_{j}(t) & \text { else }
\end{array}\right.
\end{aligned}
$$


$S(t)= \begin{cases}\sqrt{\sqrt{\sum_{t=0}^{L_{\mathrm{S}} / 2-1} z_{j}^{2}(t)}} & 0 \leq t<L_{\mathrm{S}} / 2 \\ \sqrt{\sum_{i=-L_{S} / 2}^{L_{S} / 2} z_{j}^{2}(t+i)} & L_{\mathrm{S}} / 2 \leq t<L-L_{\mathrm{S}} / 2 \\ \sqrt{\sum_{t=L-L_{\mathrm{S}} / 2}^{L-1} z_{j}^{2}(t)} & L-L_{\mathrm{S}} / 2 \leq t<L\end{cases}$

The preprocessed signal vectors $\vec{z}(t)=\left[z_{1}(t), z_{2}(t), \ldots, z_{n}(t)\right]^{\mathbf{T}}, t=0, \ldots, L-1$ whose components are shown in FIGURE 2 right are then modelled by the VAR model described in the theory section.

Signal synthesis then has to follow the process discussed so far the other way round. First the preprocessed signals $z_{j}(t)$ can be synthesized $\left(\tilde{z}_{j}(t)\right)$ with the estimated model parameters according to (1). Then spikes have to be reinserted, if desired, the differentiation has to be inverted and finally the slowly varying part of the signals has to be added. So we have

$$
\begin{aligned}
\tilde{\vec{z}}(t) & =\vec{e}(t)+\sum_{i=1}^{p} \hat{a}_{i} \tilde{\bar{z}}(t-i) \\
\bar{x}_{j}(t) & =\bar{x}_{j}(t-1)+b \tilde{z}_{j}(t) \quad b=0.7 \\
\tilde{x}_{j}(t) & =\bar{x}_{j}(t)+\hat{x}_{\mathrm{R}, j}(t)
\end{aligned}
$$

for $j=1, \ldots, n$ and $t=0, \ldots, L-1$ with $\left\{e_{j}(t)\right\}=\mathrm{N}\left(0, \hat{\Sigma}_{p, j j}\right)$.

The white noise process is assumed to have diagonal correlation matrix so it can be realized by $n$ scalar white noise processes with variances $\hat{\Sigma}_{p, j j}, j=1, \ldots, n$. If desired spikes have to be inserted in the signals $\tilde{z}_{j}(t)$ according to a protocol created during the detection and removal of them. The insertion moment of the spike is given by the protocol and the amplitude is based on a statistical model. (10) is the inversion of the differentiation process in (6). A value of 0.7 for $b$ has been found to be reasonable [6] due to the stability condition $|b|<1$ for $(10)$. To have synthesized signals $\tilde{x}_{j}(t)$ correspond to the originally recorded signals $x_{j}(t)$ it is necessary to restore the slowly varying part being lost by the differentiation procedure. This is done on the basis of a set of estimated mean values

$\hat{x}_{R, j}(k)=\frac{1}{L_{\mathrm{R}}} \sum_{i=0}^{L_{\mathrm{R}}-1} x_{j}\left(k L_{\mathrm{R}}+i\right) \quad, \quad k=0, \ldots$, int $\left(\frac{L}{L_{\mathrm{R}}}\right)-1$

which are interpolated using AKIMA's technique to obtain $\hat{x}_{\mathrm{R}, \mathrm{j}}(t)$. FIGURE 3 shows the synthesized preprocessed signals $\tilde{z}_{j}(t)$ and recorded signals $\tilde{x}_{j}(t)$. A comparison with the corresponding signals in FIGURE 2 gives good reason for the applicability of the VAR-model to these signals.

The whole modelling procedure will now briefly be summarized. The analysis (i.e. estimation part) consists of the steps: 

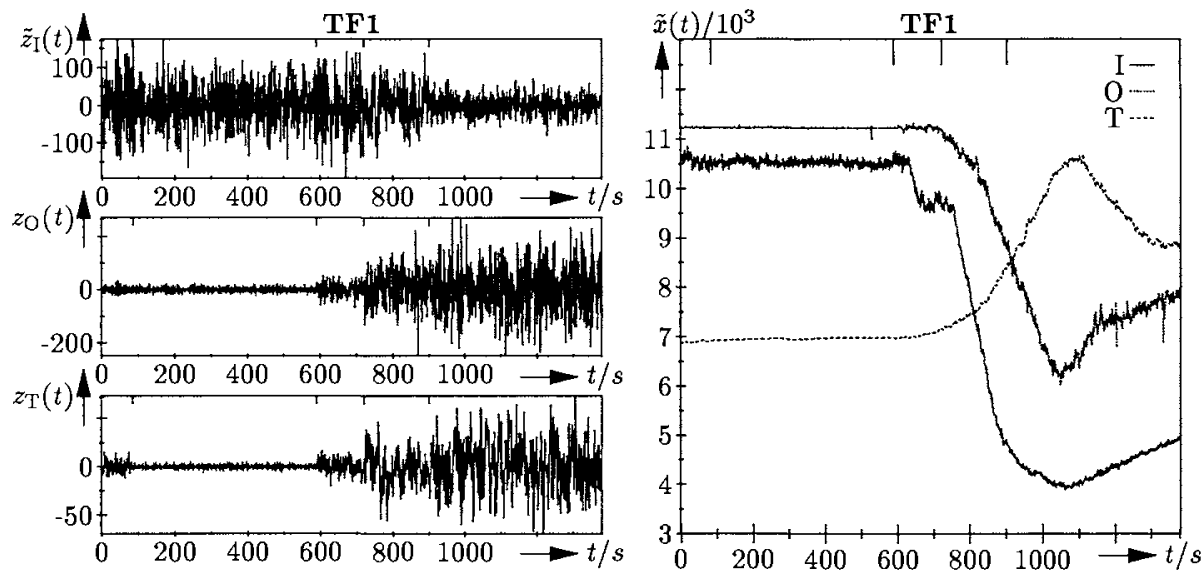

FIGURE 3: Synthesized preprocessed signals $\tilde{z}_{j}(t)\left(p_{\max }=4, L_{\text {test }}=60, D_{\text {seg }}=120\right)$ and synthesized original signals $\tilde{x}_{j}(t)\left(L_{\mathrm{R}}=30\right)$ for EN 54/9 test fire TF1. The segment boundaries are indicated by tics at the top of the windows.

1. signal preprocessing (parameters: spike window length $L_{\mathrm{S}}$ and tolerance factor $k_{\mathrm{S}}$ )

2. determination of the interpolation points $x_{\mathrm{R}, j}(k)$ for the slowly varying part of the signals (parameter: interpolation distance $L_{\mathrm{R}}$ )

3. segmentation and VAR model estimation (parameters: maximum model order $p_{\max }$, segmentation test window length $L_{\text {test }}$ and boundary detection threshold $D_{\text {seg }}$ )

4. storage of all necessary information for synthesis in a database

The signal synthesis procedure has to follow the steps:

1. synthesis of a VAR process with the model order, coefficient matrices and noise variances from the data base

2. integration of the resulting signals

3. insertion of spikes

4. addition of AKIMA-interpolated slowly varying part

\section{SIMULATION RESULTS}

In this chapter we will study the influence of the different parameters on a simple multisensor fire detection algorithm. The detection algorithm is a multi-sensor threshold detector with alarm delay. It will operate on the weighted sum

$x_{d}(t)=\frac{1}{3}\left[w_{\mathrm{I}}\left(15000-x_{\mathrm{I}}(t)\right)+w_{\mathrm{O}}\left(15000-x_{\mathrm{O}}(t)\right)+w_{\mathrm{T}} x_{\mathrm{T}}(t)\right]$

of the monitored signals. The weighted sum is a signal with positive trend in the fire case resulting from all applied sensors. For the $\mathrm{I}$ and $\mathrm{O}$ sensor the expected trend of the 


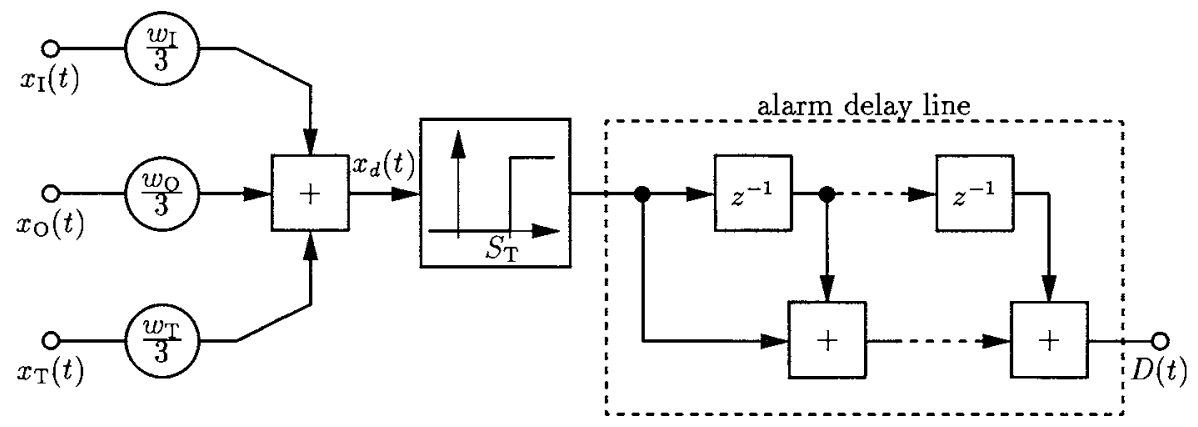

FIGURE 4: Block diagram of the 3-channel threshold detector

recorded signal in the fire case is negative and for $\mathrm{T}$ it is positive.

The detection quantity of the threshold detector (FIGURE 4) then is

$D(t)=\sum_{i=0}^{L_{\mathrm{T}}-1} \sigma\left(x_{d}(t-i)-S_{\mathrm{T}}\right)$

with $\sigma(x)=1$ for $x \geq 0$ and 0 else. With the alarm threshold $S_{\mathrm{D}}$ fixed at $L_{\mathrm{T}}-0.5$ the detector will give an alarm if the last $L_{\mathrm{T}}$ values of $x_{d}(t)$ are greater than or equal to $S_{\mathrm{T}}$. The adjustable parameters of the threshold detector are the threshold $S_{\mathrm{T}}$ and the length of the alarm delay line $L_{\mathrm{T}}$. The following parameter combinations will be studied: $S_{\mathrm{T}}=0.6,0.7,0.8,0.9$ and $L_{\mathrm{T}}=30,60,90,120$. The weights $w_{\mathrm{I}}, w_{\mathrm{O}}$ and $w_{\mathrm{T}}$ in (13) were set to $10^{-4}$ during all simulations.

The following figures show the results obtained with a simulation based on the VAR model with the adaptions discussed in the previous section. The simulation study of this example detector will treat the fire case based on recordings of the test fires according to EN 54 part $9[8]$ and the non-fire case based on recordings from two false alarm relevant installations (a hospital and a steel work).

The parameters of interest are the detection times and rates for the 6 test fires and estimates of the false alarm rate. The database for the fire case consists of 9 recordings of each test fire. 20 runs over the database lead to 180 simulations each for the test fires. In the non-fire case the database contains a total of 2 years of signal recordings over a total observation time of 12 years from different locations at the hospital and the steel work.

FIGURE 5 shows the distribution of the maximum of $x_{d}(t)$ during the simulations in the fire case for each of the test fires. Combined with the distribution of $x_{d}(t)$ in the non-fire case (FIGURE 6 left) obtained through one run over the whole non-fire database the above made choice of the thresholds $S_{\mathrm{T}}$ becomes clear.

For these values of $S_{\mathrm{T}}$ and the delay lengths $L_{\mathrm{T}}$ the detection rate $n_{\mathrm{d}}$ in $\%$ and the average detection times $t_{\mathrm{D}}$ are shwon in figure FIGUREs 7 and 8 . A detection time of 

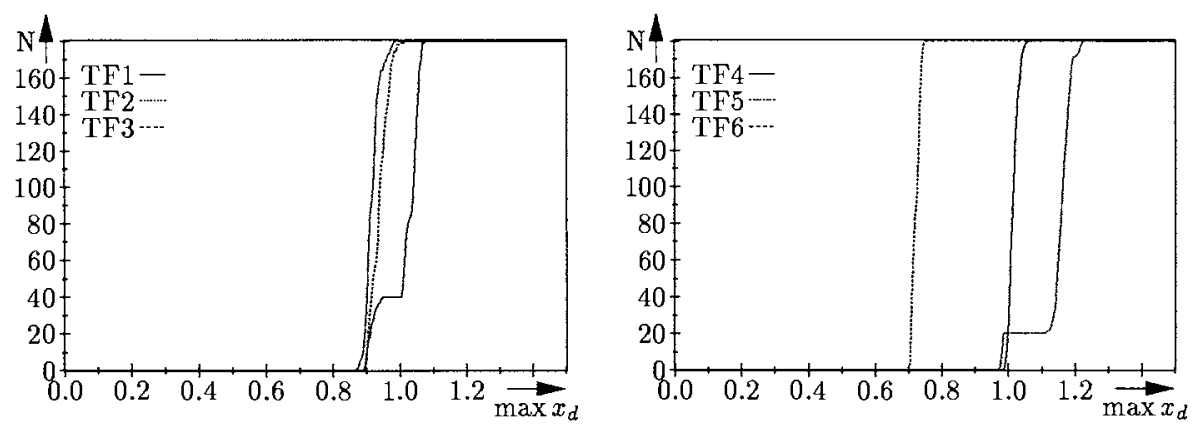

FIGURE 5: Distribution of maximum $x_{d}(t)$ for the 180 simulations of each of the six test fires TF1 to TF6.

0s corresponds to no detection of the fire. For the calculation of the detection times only the detected fires are used. The corresponding false alarm rate estimates $\hat{\alpha}$ (simulation time 15 years) are shown in FIGURE 6 right.

In general the behaviour of the average detection time $t_{\mathrm{D}}$ in FIGURE 7 is straight forward since we have a linear detection algorithm (14). The poor detection of TF2 $\left(S_{\mathrm{T}}=0.9\right)$ and the loss of TF6 $\left(S_{\mathrm{T}}=0.8,0.9\right)$ in FIGURE 8 shows the problems of this detection principle. Furthermore the detection times of TF3 $\left(S_{\mathrm{T}}=0.8,0.9\right)$ and TF2 $\left(S_{\mathrm{T}}=0.9\right)$ (FIGURE 7) are unacceptaple long.
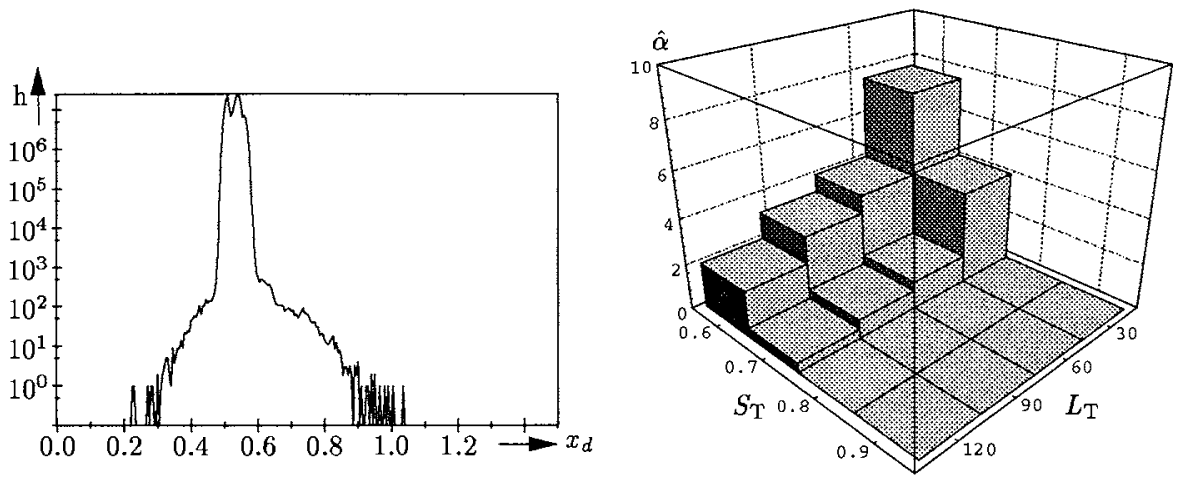

FIGURE 6: Distribution of $x_{d}(t)$ in nonfire situations (left) and estimated false alarm rates $\hat{\alpha}$ per year for the threshold detector (right). 
TF1



TF3

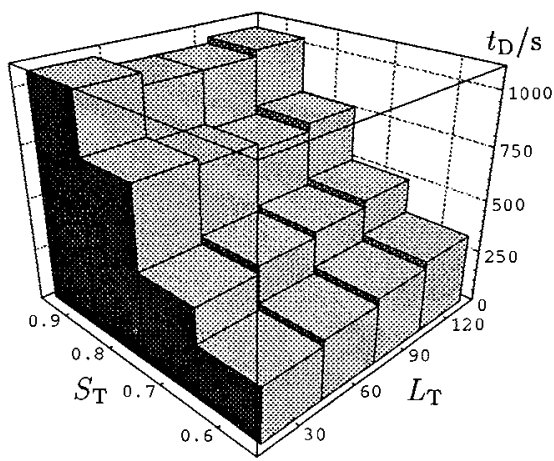

TF5

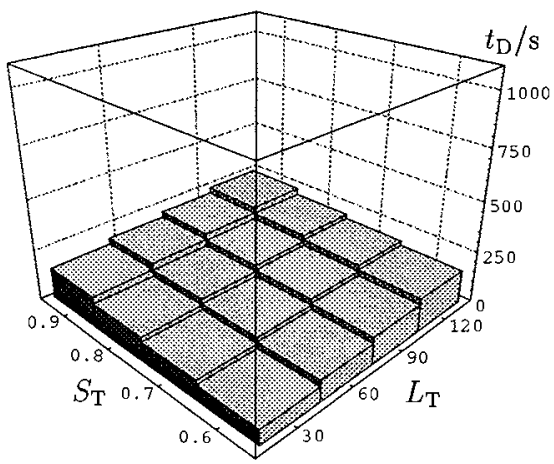

TF2

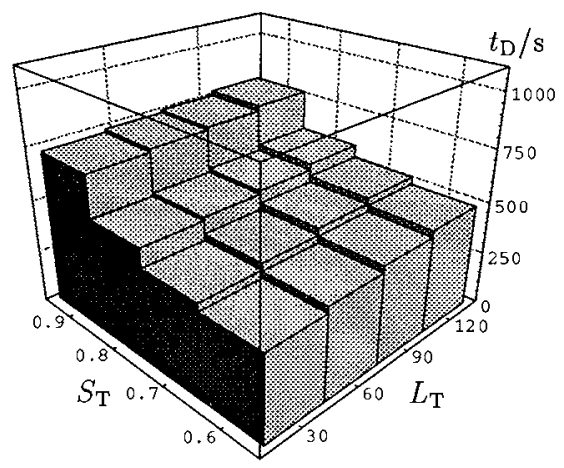

TF4

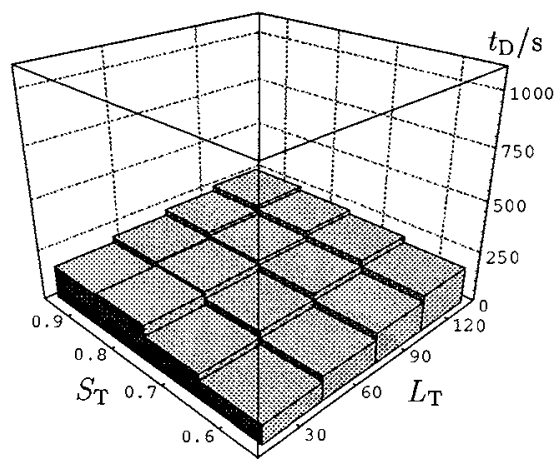

TF6

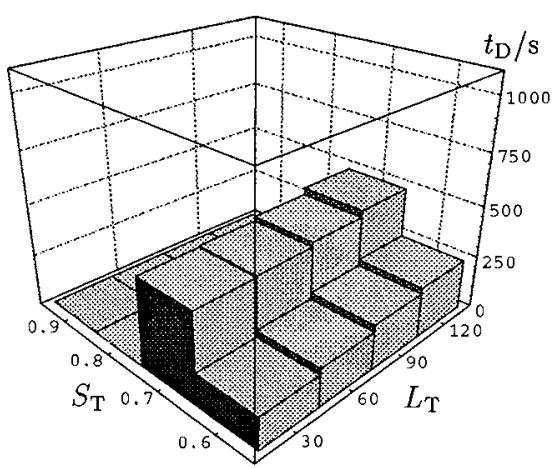

FIGURE 7: Detection times of EN 54/9 test fires for the 3-channel threshold detector 
TF1

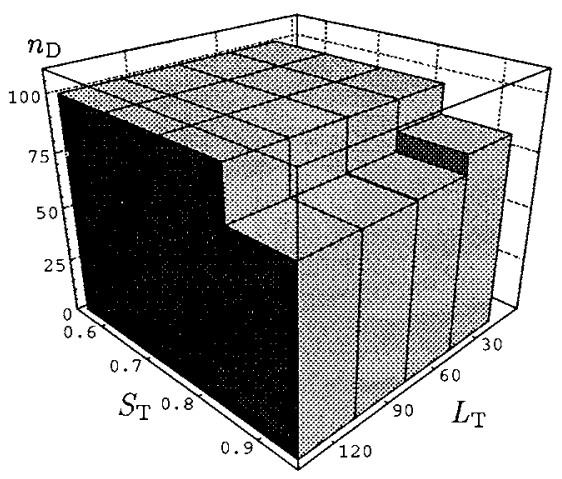

TF3

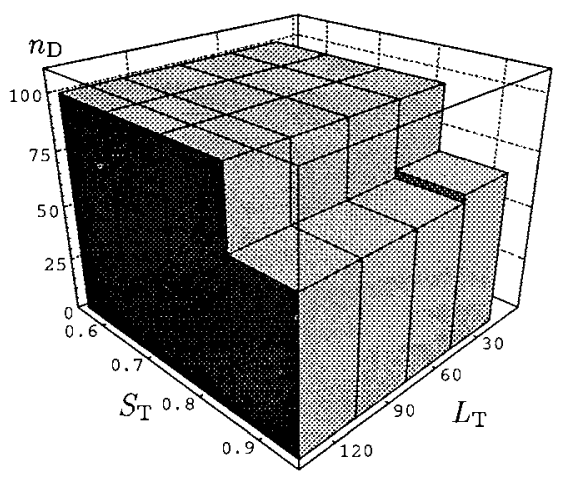

TF5

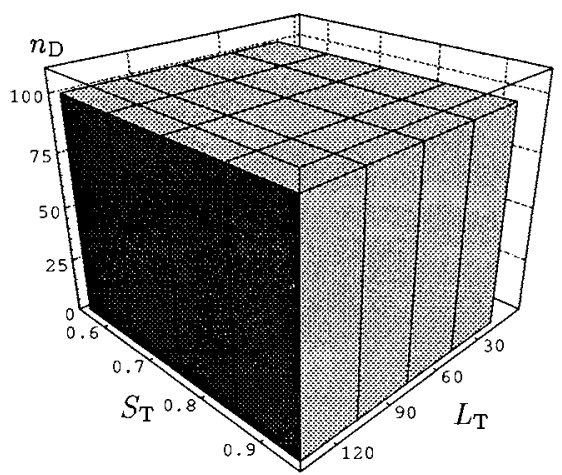

TF2

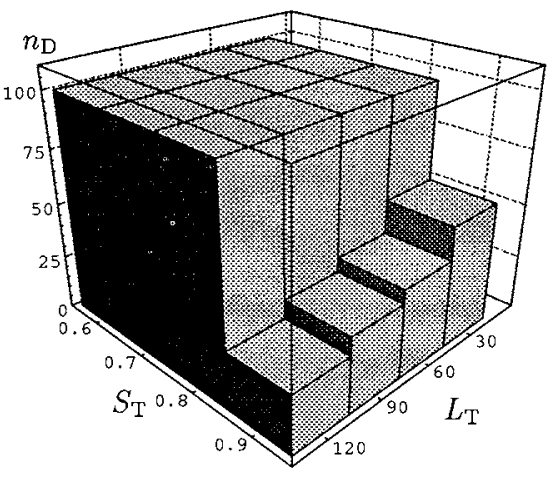

TF4

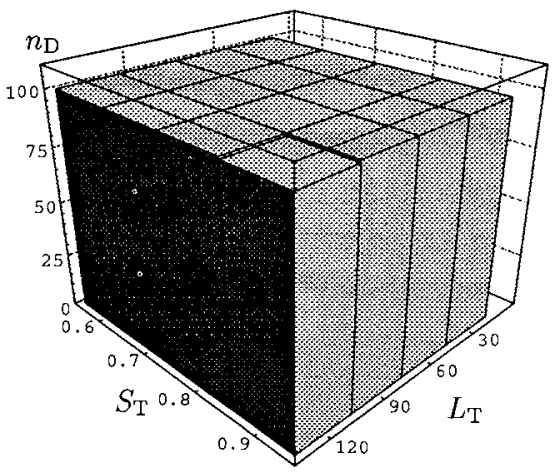

TF6

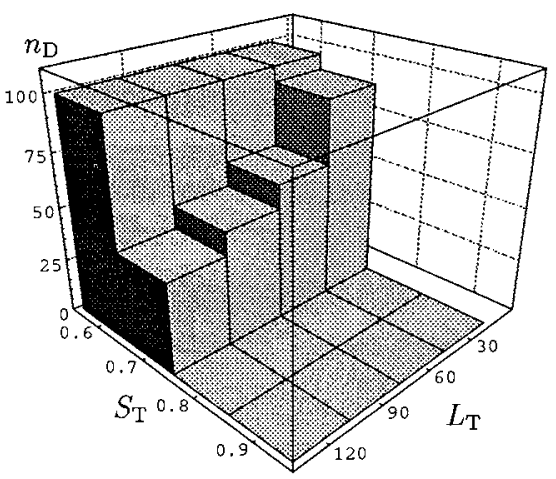

FIGURE 8: Percentages of detected fires for the 3-channel threshold detector 


\section{CONCLUSION}

The VAR model was presented as a model for non-stationary multi-channel discrete-time processes. The necessary extensions for the modelling of fire signals were described. An application of the simulation technique to a simple multi-sensor fire detection algorithm focussing on the detection capability was given. The simulation results give quantitative estimates for the detection time and rate and the false alarm rate of the algorithm for various parameter combinations. The simulation technique hence is well applicable to comparative studies of multi-sensor fire detection algorithms.

\section{REFERENCES}

[1] Brockwell, P. J. and Davis, R. A., Time Series: Theory and Methods, Springer Verlag, New York, 2nd edition, 1991.

[2] Lütkepohl, H., Introduction to Multiple Time Series Analysis, Springer, Berlin, 1991.

[3] Strobach, P., Linear Prediction Theory: A Mathematical Basis for Adaptive Systems, Springer, Berlin, 1990.

[4] Wei, W. S., Time Series Analysis - Univariate and Multivariate Methods, Addison Wesley, Redwood City, California, 1990.

[5] Appel, U. and v. Brandt, A., "Adaptive Sequential Segmentation of Piecewise Stationary Time Series", Information Sciences, 29, 27-56, 1983.

[6] Klose, J., Ermittlung der Eigenschaften von Signaldetektoren durch Analyse und Simulation von Zufallsprozessen mit einer Anwendung in der automatischen Brandentdeckungstechnik, $\mathrm{PhD}$ thesis, Universität-Gesamthochschule-Duisburg, 1992.

[7] Sokat, J., Ein Beitrag zur adaptiven Filtertheorie mit einer Anwendung in der automatischen Brandentdeckungstechnik, $\mathrm{PhD}$ thesis, Universität-GesamthochschuleDuisburg, 1991.

[8] CEN European Committee for Standardization, EN 54 part 9, Components of automatic fire detection systems: Fire sensitivity test, Brussels, 1984. 